\title{
Maternal Autonomy, Distance to Health Care Facility and ANC Attendance: Findings from Madiany Division of Siaya County, Kenya
}

\author{
C.O. Asweto ${ }^{1,2, *}$, J.R. Aluoch ${ }^{1}$, C.O. Obonyo ${ }^{3}$, J.O. Ouma ${ }^{4}$ \\ ${ }^{1}$ School of Public Health and Community Development, Maseno University, Kisumu, Kenya \\ ${ }^{2}$ Faculty of Health Science, Great Lakes University of Kisumu, Kisumu, Kenya \\ ${ }^{3}$ Kenya Medical Research Institute (KEMRI), Kisumu, Kenya \\ ${ }^{4}$ Public Health Department, University of Kabianga, Kabianga, Kenya \\ *Corresponding author: aswetocollins@yahoo.com
}

Received June 09, 2014; Revised July 29, 2014; Accepted August 11, 2014

\begin{abstract}
Half a million women die annually from pregnancy related causes. In Kenya, maternal mortality ratio (MMR) remains high in spite of great efforts to improve maternal health care. We studied some factors linked to maternity in a rural community setting. A survey was conducted in the community living in Madiany division of Siaya County, Kenya. We randomly sampled 403 mothers of children under three years old. Variables of interest were antenatal care attendance (ANC), parity, distance from the health facility and women empowerment (autonomy). Nearly $94 \%$ of the mothers had at least one ANC visit and $48.6 \%$ had completed the recommended four or more ANC visits; about $65.7 \%$ initiated ANC at first trimester. Parity, distance to the health facility and autonomy were associated with ANC visits. Mothers with one child had a twice higher chance of at least four ANC visits than those with more than one child. Those living at most 60 minutes travel-time from the health facility were seven times more likely have early ANC initiation and five times more likely to have at least four ANC attendance than those living more than 60 minutes travel-time from the health facility. Autonomy was linked to early initiation of ANC visit. This may indicate that improving maternal care and achieving Millennium Development Goal (MDG) 5 target, it is important to improve coverage of health facilities and implement development programs that empower rural women.
\end{abstract}

Keywords: antenatal care, maternal mortality ratio, autonomy

Cite This Article: C.O. Asweto, J.R. Aluoch, C.O. Obonyo, and J.O. Ouma, "Maternal Autonomy, Distance to Health Care Facility and ANC Attendance: Findings from Madiany Division of Siaya County, Kenya." American Journal of Public Health Research, vol. 2, no. 4 (2014): 153-158. doi: 10.12691/ajphr-2-4-5.

\section{Background}

Globally, more than half a million women die every year due to pregnancy related causes, with $99 \%$ of these in developing countries [1]. In the year 2005, WHO estimated maternal mortality ratio of 900/100,000 live births for Sub-Saharan Africa with a lifetime risk of a maternal death of 1 in 22 . These rates are high when compared to the developed countries where the lifetime risk of 1 in 7300 was estimated during the same time period [2].

The Kenyan government has paid great attention to maternal health care including development of strategies to improve access to primary health care [3]. Despite of these, maternal mortality ratio (MMR) still remains high. The Kenya Demographic and Health Survey (KDHS) documented a maternal mortality ratio (MMR) of $488 / 100,000$ live births [4]. The life time risk of a maternal death in Kenya has been estimated at 1 in 39 [2]. According to the $2008 / 2009$ KDHS, more than nine in ten mothers reported seeing a health professional at least once during pregnancy for antenatal care. The above national scenario was also reflected in Nyanza province where $93.6 \%$ of pregnant women sought professional antenatal care [4].

Direct causes of maternal mortality include hemorrhage (34\%), infections $(21 \%)$, hypertensive disorders (16\%), and obstructed labour (11\%) [5]. These complications could be prevented, if women received appropriate care at critical times of pregnancy and delivery. This is the rationale for implementing maternity services, which aims to screen women for signs or risk factors which may potentially lead to obstetric complications with the goal of providing appropriate effective interventions. Antenatal care provides an opportunity to identify and treat the danger signs, in order to guarantee a healthy mother and baby at the end of a pregnancy. Antenatal and delivery care are an effective way to reduce maternal mortality in developing countries. However, maternal health care system in developing countries has been adapted from developed countries without formal evaluations of the efficacy of interventions in developing country settings [1] 
Maternal health care services are available but their use is low in Kenya [3]. Currently, it is unclear why most women do not use maternal health care services. Even in urban areas where physical accessibility is not considered a major constraint, about one-fourth of all deliveries still occur away from health facilities [3]. A study done in rural western Kenya showed that use of ANC was high, but the opportunity to deliver in health facilities was not fully utilized [6]. Use of professional delivery services was low, and almost 1 out of 5 women delivered unassisted $^{6}$. This finding indicates that a significant proportion of women fail to seek delivery by trained health workers. The risk of complications and deaths are highly increased under such situations. This is attributed to by lack of adequate data on factors contributing to this phenomenon, which hampers the development of appropriate health interventions and responsive strategies especially in rural areas.

There exists an association between the use of antenatal care and positive maternal outcomes [21]. Antenatal care alone cannot prevent all obstetric emergencies. However, the information provided by antenatal service providers is important for the successful management of pregnancies and the subsequent wellbeing of the child. Antenatal care provides an entry point for women to the health care system. The objective of the system is to monitor the pregnant women regularly during their pregnancy, so that risk factors for adverse outcome can be identified [22]. Antenatal care presents an opportunity to evaluate the mother's overall condition, diagnose and treat infections, screen for anaemia and HIV/AIDS and prevent low birth weight [23].

Other potential benefits of antenatal care are counseling on nutrition and healthy pregnancy/delivery behavior; provide tetanus immunization, malaria prophylaxis, iron and folic acid supplementation and assist women to select a trained birth attendant or institution to deliver their babies in. About a half of all women in developing countries make at least four antenatal care visits during pregnancy [23]. However, there are a number of factors in developing countries that act as hindrances to these aspects of antenatal care from being successful. Therefore, the purpose of this study was to determine factors influencing the antenatal care utilization among women of Madiany Division of Rarieda district, western Kenya. Thus contributing to developing interventions that serve as an important tool for improving the use of maternal health care services.

\section{Methodology}

\subsection{Study Site}

This study was conducted in Central Uyoma location, Madiany division of Rarieda district in Siaya county, Kenya. The predominant tribe is Luo who earn their living through subsistence farming and fishing. The Location has three sub-locations namely, Kobong', Rachar and Masala, with a total of 35 villages. In 2009 census, the location was recorded to have a total population of 13,830 people [4]. The district experiences high rates of maternal mortality, estimated at 620 deaths per 100,000 live births [7].

\subsection{Study Design}

A cross-sectional study design was adopted to enable collection of information from a large population over a short period of time. It also allowed the study to be carried out in the natural real-life settings using samples, thus increasing the external validity (generalizability) of the study.

\subsection{Study Population}

The study population was women of reproductive age (15-49 years) who were residents of Central Uyoma location, Madiany Division of Rarieda district and the sampling unit was the households within the location.

\subsection{Sample Size Determination}

In order to detect a minimum difference of $10 \%$ with power of $80 \%$ and $95 \%$ confidence, a minimum sample size of 194 was required. This was adjusted for an anticipated non-response rate of $5 \%$ and hence the total minimum sample size was 202. This was doubled to maintain a minimum sample of 402 .

\subsection{Sampling Procedure}

A total of thirty one villages were selected from the division using multistage random sampling with population proportion to size (PPS). Locations were listed and selected in the first stage using simple random sampling, and villages within selected location were selected using simple random sampling in the second stage. Individual households within villages were selected to identify women of reproductive age who participated in the study.

\subsection{Data Collection}

Data was collected using interviewer-administered structured questionnaire. Information on sociodemographics characteristic, utilization of maternal health care, maternal knowledge on pregnancy and delivery danger signs, and health facility characteristics were collected.

\subsection{Recruitment and Training of Research Assistants}

Six lady residents, who had graduated from high school with a mean grade of at least $\mathrm{C}$ - and spoke both English and Luo (the local oral dialect) fluently, were recruited as research assistant to administer questionnaires. Ladies were chosen because; women could easily share of their pregnancy and delivery experience with their fellow ladies. They were also trained for three days on using the tool by the researcher. This was done to ensure quality is maintained in data collection.

\subsection{Data Management, Analysis and Presentation}

The collected data was in the custody of the researcher who always ensured privacy and confidentiality of the information given by the respondents. The data was later entered in Statistical Package of Social Science (SPSS for 
Windows 16.0 SPSS Inc. Chicago Illinois) software. Logistic regression was done in which odds ratios with 95\% confidence intervals were calculated to determine association between independent and dependent variable. The data were presented in tables.

\subsection{Ethical Considerations}

This study commenced after approval had been received from Maseno University, School of Public Health and Community Development and School of Graduate Studies. The research permit was sought from National Council for Science and Technology (Kenya). Permission was also sought from local administration in advance before data collection. The study participants made a verbal informed consent prior to inclusion in the study.

\subsection{Measurement of Variables}

\subsubsection{Dependent Variable}

Maternal health care was the dependent variable in this study. In the study, the respondents (women aged 15-49 years) were asked whether, with respect to last pregnancy and live birth occurring in the last three years they had any antenatal care. Dichotomous dependent variables were created from this maternal health specific questions, namely: (a) If the women had received antenatal cares check up at least once during their pregnancy from formal sources; $1=$ received antenatal care from doctors, nurses, midwives; $0=$ received antenatal care from TBA, other informal sources, none at all. (b) The time women initiated their antenatal care visit; $1=$ at first trimester; $0=$ at second and third trimester. (c) The number of antenatal care visits the women had during their last pregnancy; $1=$ had more than four visits; $0=$ had less than four visits.

\subsubsection{Measurement of Independent Variable}

\section{Maternal characteristics}

Age of women: The question on the age of the women at the time of survey included the date of birth.

Marital status: Married where those who were in marriage life at the time of the survey, while widowed, separated, divorced and never married were considered as single.

Women's Education: Maternal education was categorized into four classes namely; no education, primary (completed class 8), secondary (completed form four) and tertiary education (above secondary school). These categories were made to allow for nonlinearity in the effects of education on utilization behavior and correspondent to primary, secondary and tertiary education as is currently prevalent in KDHS [4].

Women's Employment Status and Women Occupation: The measurement of women's employment was only considered for those women who were currently employed, i.e. performed some work, at least one hour in the week prior to the survey, for wage/salary, or profit or family gain in cash or in kind [20]. Those who were not currently employed but had worked in the past 12 months are not included in the sample.

Women's Autonomy: The degree of women's autonomy was captured in one area; her decision making power. The research questions the respondents on the following issues. Firstly, who decided on use antenatal acre services? The decisions were categorized as follows: $1=$ Self or self and husband or self and others; $2=$ Husband or others.

Parity: In this study the categorization of birth order was classified into four categories: those who had one child; those who had 2-3 children; those who had 4-6 children and those who had 7 children or more.

Knowledge of women about danger signs of pregnancy/delivery: This variable was categorized into three levels. High knowledge applies to those who know at least $60 \%$ of danger signs of pregnancy and or delivery. Low knowledge refers to the respondents who know less than $60 \%$ dangers of pregnancy and delivery, while No knowledge refers to those who do not know 30 any danger sign of pregnancy and delivery. Knowledge is coded as High $=2$, Low $=1$ and No $=0$.

Health care center's characteristics:

Travel time: This variable indicates the travel time from a woman's home to a health facility.

Perceived quality of care: This variable indicates the perception of women on quality of health care in the health facility and was coded as (excellent, good, fair and poor).

\section{Results}

The age of the respondents ranged from 15 to 48 years, with a mean age of $26.6 \pm 6.7$ years. About $182(45.1 \%)$ women fell in the 15-24 years age. The monthly family income of respondents ranged from Kshs. 500 to Kshs. 45,000 with a mean income of Kshs. 4,699.06 per month. About 129 (31.9\%) were housewives, while 132 (32.8\%) were business women. Most of the respondents, 243 $(60.3 \%)$ had completed primary education and $323(80.1 \%)$ had at least two children. Table 1 is a summary of their demographic characteristics.

Table 1. Socio-demographic characteristics of respondents

\begin{tabular}{|c|c|}
\hline Characteristics & Number $(\%)$ \\
\hline \multicolumn{2}{|l|}{ Age (years) } \\
\hline $15-24$ & $182(45.1)$ \\
\hline $25-34$ & $161(40.0)$ \\
\hline $35-44$ & $55(13.7)$ \\
\hline $45+$ & $5(1.2)$ \\
\hline \multicolumn{2}{|c|}{ Monthly income (Kshs.) } \\
\hline$<5000.00$ & $148(70.4)$ \\
\hline$\geq 5000.00$ & $62(29.6)$ \\
\hline \multicolumn{2}{|l|}{ Education } \\
\hline None & $120(29.7)$ \\
\hline Primary & $243(60.3)$ \\
\hline Secondary & $33(8.3)$ \\
\hline Tertiary & $7(1.7)$ \\
\hline \multicolumn{2}{|l|}{ Working status } \\
\hline Employed & $26(6.4)$ \\
\hline Farmer & $84(20.8)$ \\
\hline Businesswoman & $132(32.8)$ \\
\hline House wife & $129(31.9)$ \\
\hline Others (e.g. student) & $32(6.1)$ \\
\hline \multicolumn{2}{|l|}{ Parity } \\
\hline 1 & $80(19.9)$ \\
\hline $2-3$ & 169 (41.9) \\
\hline $4-6$ & $131(32.4)$ \\
\hline $7+$ & $23(5.9)$ \\
\hline
\end{tabular}




\subsection{Utilization of Antenatal Care Services}

Majority, 378 (93.8\%) of the respondents had at least one antenatal visit during their last pregnancy. Among them, $196(51.9 \%)$ had four or more visits during their last pregnancy, whereas $182(45.2 \%)$ had less than four ANC visits. About 248 (65.6\%) respondents started their antenatal care visit during the first trimester Table 2 summarizes the level of maternal health care utilization.

Table 2. Level of antenatal care utilization

\begin{tabular}{lll}
\hline Characteristics & Number & Percentage (\%) \\
\hline $\begin{array}{l}\text { ANC visit during the last pregnancy } \\
\text { Yes }\end{array}$ & 378 & 93.8 \\
No & 25 & 6.2 \\
Number of ANC visits & & \\
$<4$ & 182 & 48.1 \\
$\geq 4$ & 196 & 51.9 \\
Timing of ANC visit initiation & & \\
$1^{\text {st }}$ trimester & 248 & 65.6 \\
$2^{\text {nd }}$ trimester & 123 & 32.5 \\
$3^{\text {rd }}$ trimester & 7 & 1.9 \\
\hline
\end{tabular}

3.2. Maternal Characteristics and Utilization of Antenatal Care
As shown in Table 3, maternal age, maternal knowledge on danger signs pregnancy and delivery, maternal education and working status of women were found to have no significant association with ANC use, time of initiating ANC visit and number of ANC visits. However, parity was significantly associated with number of ANC visits and mothers with one child were almost twice more likely to have at least four ANC visits than mothers with more than one child $[\mathrm{OR}=1.75(1.06-2.93)]$. Women with autonomy were six times more likely to have at least one ANC attendance $[\mathrm{OR}=5.95(2.4-8.19)]$ and 2.5 times more likely to have early ANC initiation $[\mathrm{OR}=2.50$ (1.48-4.23)] than women without autonomy.

\subsection{Health Facility Characteristic and Utilization of Antenatal Care Services}

As shown in Table 4, mothers who travel less than one hour had seven times more likely to have early ANC initiation $[\mathrm{OR}=6.91(1.72-12.1)]$ and five times more likely to have at least 4 ANC visits than mothers who travel more than one hour $[\mathrm{OR}=4.98$ (3.26-7.96)]. Women perception on quality of care was found to have no significant relationship with ANC use, early initiation of ANC visit and having at least four ANC visits. Perceived quality of care had no influence on antenatal care services.

\begin{tabular}{|c|c|c|c|c|c|c|}
\hline \multirow[b]{2}{*}{ Variable } & \multicolumn{2}{|l|}{ ANC use } & \multicolumn{2}{|c|}{ ANC Initiation at 1ST Trimester } & \multicolumn{2}{|c|}{$\geq 4$ ANC visits } \\
\hline & Freq $(\%)$ & OR $(95 \% \mathrm{CI})$ & Freq $(\%)$ & OR $(95 \% \mathrm{CI})$ & Freq $(\%)$ & OR $(95 \%$ CI $)$ \\
\hline \multicolumn{7}{|l|}{ Age } \\
\hline$\leq 24$ years & $167(93.5)$ & $0.53(0.21-1.33)$ & $116(64.1)$ & $1.18(0.79-1.76)$ & $95(56.0)$ & $1.34(0.91-1.99)$ \\
\hline$>24$ years & $211(96.4)$ & 1 & $132(60.3)$ & 1 & $101(48.7)$ & 1 \\
\hline Educational level & & 1 & & & & \\
\hline None & $104(92.9)$ & $0.441(0.16-1.21)$ & $67(58.4)$ & 1 & $57(53.1)$ & 1 \\
\hline Primary & $236(96.7)$ & $1.04(0.26-4.11)$ & $157(63.4)$ & $1.23(0.78-1.95)$ & $133(57.3)$ & $1.40(0.90-1.95)$ \\
\hline$\geq$ Secondary & $38(92.7)$ & & $25(61.0)$ & $1.11(0.54-2.31)$ & $26(68.3)$ & $1.90(0.92-3.94)$ \\
\hline \multicolumn{7}{|l|}{ Knowledge } \\
\hline High & $43(95.6)$ & $1.12(0.21-6.00)$ & $14(31.1)$ & $1.27(0.59-2.74)$ & $18(40.0)$ & $1.19(0.58-2.44)$ \\
\hline Low & $98(95.1)$ & 1 & $27(26.2)$ & 1 & $37(35.9)$ & 1 \\
\hline \multicolumn{7}{|l|}{ Work status } \\
\hline Working & $229(95.9)$ & $1.52(0.63-3.74)$ & $157(64.1)$ & $1.25(0.81-1.82)$ & $123(51.0)$ & $0.91(0.61-1.35)$ \\
\hline Not working & $149(93.9)$ & 1 & $88(35.9)$ & 1 & $73(53.4)$ & 1 \\
\hline \multicolumn{7}{|l|}{ Parity } \\
\hline 1 Child & $73(92.6)$ & $0.56(0.21-1.50)$ & $54(67.9)$ & $1.38(0.82-2.31)$ & $51(63.0)$ & $1.75(1.06-2.93)$ \\
\hline$>1$ Child & $305(95.7)$ & 1 & $194(60.6)$ & 1 & $16(49.2)$ & 1 \\
\hline \multicolumn{7}{|l|}{ Autonomy } \\
\hline Autonomy & $328(99.4)$ & $5.95(2.4-8.19)^{*}$ & $221(65.8)$ & $2.50(1.48-4.23)^{*}$ & $157(46.3)$ & $0.66(0.39-1.11)$ \\
\hline No-autonomy & $50(73.9)$ & 1 & $27(43.5)$ & 1 & $39(56.5)$ & 1 \\
\hline
\end{tabular}

Table 4. Health facility factors associated with antenatal care utilization

\begin{tabular}{|c|c|c|c|c|c|c|}
\hline \multirow[b]{2}{*}{ Variable } & \multicolumn{2}{|l|}{ ANC use } & \multicolumn{2}{|c|}{ ANC Initiation at 1ST Trimester } & \multicolumn{2}{|c|}{$\geq 4$ ANC visits } \\
\hline & Freq $(\%)$ & OR $(95 \%$ CI $)$ & Freq $(\%)$ & OR $(95 \% \mathrm{CI})$ & Freq $(\%)$ & OR $(95 \%$ CI $)$ \\
\hline \multicolumn{7}{|c|}{ Travelling time } \\
\hline$\leq 60$ minutes & $238(98.8)$ & $1.03(1.00-107)$ & $193(80.1)$ & $6.91(1.72-12.1)$ & $163(68.5)$ & $4.98(3.26-7.96)$ \\
\hline$>60$ minutes & $121(96.8)$ & 1 & $46(36.8)$ & 1 & $33(30.4)$ & 1 \\
\hline \multicolumn{7}{|c|}{ Perceived quality of are } \\
\hline Good & $282(99.3)$ & $2.96(0.41-21.3)$ & $185(64.7)$ & $0.93(0.69-1.31)$ & $154(54.5)$ & $1.02(064-1.62)$ \\
\hline
\end{tabular}

pregnancy [13]. In this study, the overall ANC attendance

\section{Discussion}

World Health Organization recommends a minimum of four ANC visits initiated during the first trimester of during the previous pregnancy was found to be high $93.8 \%$. However, slightly more than six out of ten women initiated their first ANC visit at first trimester. Moreover, a significant proportion $(48.1 \%)$ of the respondents had 
made less than four visits which is less than the recommended. This shows low utilization of antenatal care services. This finding is consistent with the result of the survey conducted by KDHS in 2008/2009 which showed $93.6 \%$ attendance of ANC in Nyanza province and about four out of ten women delivered in health facility [4].

Possession of correct knowledge is the first factor that affects attitude, intention, and behavior. It relates to behavior, which may produce attitudinal change toward service utilization ${ }^{8}$. The higher level of knowledge about the importance of danger signs that women experience, the more likely were women to attend antenatal care [1]. In this study knowledge about the danger signs of pregnancy and delivery had no association with antennal care use. This is contrary to previous studies which have emphasized the importance of raising awareness among women of reproductive age, especially among the uneducated $[9,10,11]$. However, improving knowledge about the benefits of antenatal care for pregnant women is an important element in enabling them to enrich their experiences as well as supporting their effort to better appreciate ways to protect their health and that of their unborn babies.

This study revealed that parity influenced four ANC visits. As the number of children a woman have increases, utilization of ANC becomes less likely. Interestingly, association between maternal age, maternal education, and maternal occupation with ANC service utilization was not statistically significant. This finding is contrary to the findings of previous study conducted in Addis Ababa [12] which showed that maternal education, maternal age and maternal occupation were strong determinants of ANC service utilization. However, a study by Chakraborty et al. (2003) also found that the age of a woman had no impact on utilization of maternal health care. Develay et al., (1996) argues that if a relationship does exist, the association between utilization of maternal health care and age women is often very weak. Even though those studies were carried out in urban areas where all women tend to use not only maternal health care services but also other health services [13], this study was done in rural area where ANC uptake was almost $100 \%$, thus age, education and occupation had no influence in the uptake.

Magadi et al. (2000) also found no relationship between the education of women and service utilization using a multivariate analysis of data from women of different communities in Kenya. The authors discuss that there is variability, at the community level, that influences the frequency of antenatal care visit during pregnancy [14]. Similarly in this study education level of women had no relationship with the utilization of antenatal care services through, the use of antennal care services was uniform across the board whether educated or not.

Employment can increase women's economic autonomy and reproductive health status. This may be because it raises their awareness and provides new ideas, behavior and opportunities through interaction with other people outside the home and community [15]. In this particular study, working status of women had no influence on the antenatal care services. Even though working women were more likely to use of antenatal care, but it was not statistically significant. Magadi et al. (2000) reported that in Kenya women in paid employment were more likely to have greater knowledge about pregnancy and childbirth due to freedom of movement outside the homestead. They also tend to seek information on services available for pregnancy care during work [14].

The influence of women's autonomy on the use of health care appears to be important. Women's autonomy can be conceptualized as their ability to determine events in their lives, even though men and other women may be opposed to their wishes [16]. This study found that autonomous women were more likely to initiate antennal care in the first trimester. In fact, autonomy was found to significantly influence utilization of maternal health care services. Bloom et al. (2001) found that dimensions of autonomy such as freedom of movement, decision making power and control over finances exert a strong influence over service use and service choice in a South Asian setting [16]. In a North Indian City, women's autonomy, as measured by the extent of a women's freedom of movement, appears to be a major determinant of maternal health care utilization among the poor to middle income women [16]. Therefore, women's autonomy is an important factor to consider in determining antenatal care use.

Average time taken to reach health facility had influence in the use of antenatal care. Women who take shorter time to reach a health facility were more likely to use antenatal care services compared to women who resided further away. A similar conclusion was reached by other study showing that long distances to a health facility caused reduction in accessibility to maternal health care services [14]. In rural areas, a lack of access roads to villages poses a serious problem, indicating that alternative approaches should be developed to better protect women's health.

When women are assured of quality of care during pregnancy, then most maternal deaths can be prevented. However, in this study women's perceived quality of care had no statistical significant to the utilization of antenatal care services. Contrary to this, a study in Namibia found that, perceived low quality of health services in the health facilities is correlated with increases maternal morbidity and mortality [17]. According to Fotso et al.(2006) the influence of women's perceived quality of care is strong in the expected direction for women with a high perception, who are more likely to deliver in an appropriate health facility compared to their counterparts with middle or low perception.

\section{Conclusion}

There is high percentage of women having at least one ANC visits, however only two thirds had early initiation and a handful of them had at least four ANC visits as recommended by WHO. This indicates low utilization of antenatal care services. Maternal autonomy, parity and distance to health facility were predicting the pattern of antenatal care utilization.

\section{Recommendations}

To ensure adequate utilization of antenatal care there is need to improve coverage of health facilities which 
provide skilled health care services by use of mobile clinics to provide services to remote villages, and health professionals should make home-care visits to pregnant women in remote communities.

There is need to design intervention targeting women of higher parity in order to improve on number of ANC visits as recommended by WHO.

Improved level of women education in rural should be encouraged, as this will reduce their dependence in matters of meeting their health needs, including maintaining their health during pregnancy and childbirth.

\section{Limitations of the Study}

Despite the contribution of the study to the literature on maternal health care, this study has some limitations. First, it is a cross-sectional study in which temporal relations could not be assessed. Second, there could be recall bias since the women were asked for events within the last three years prior to the survey despite the fact that, the most recent births were considered. Third, the study has considered only delivery of last child; the situations for deliveries of other children are unknown.

\section{Acknowledgements}

The author would like to thank Maseno University for sponsoring the research work. Also like to thank all respondents for their willingness to participate in the study. Without whose consent this research work would not have come into existence.

\section{References}

[1] WHO and UNICEF. (2003). Antenatal care in developing countries. Promises, achievements and missed opportunities, An analysis of trends, levels and differentials, 1990-2001. Geneva: World Health Organization.

[2] WHO, UNICEF, UNFPA and World-Bank. (2007). Maternal Mortality in 2005: Estimates developed by estimates developed by WHO, UNICEF, UNFPA, and the World Bank. Geneva: World Health Organization.

[3] MOH. (2006). Health service availability and utilization. Retrieved 22 January, 2011. from http://www.health.go.ke.

[4] KNBS. (2010). Kenya Demorgraphic Health Survey 2008/2009.

[5] WHO. (2001). Women's health in South-East Asia: Women Health profile factors determining women's health.: WHO regional office for south east Asia.
[6] van Eijk, A. M., Bles, H. M., Odhiambo, F., Ayisi, J. G., Blockland, I. E., Rosen, D. H., Adazu, K., Slusker, L. and Lindblade, K. A. (2006). Use of antenatal services and delivery care among women in rural western Kenya. Reproductive Health, 3(2).

[7] RDDP. (2009). Rarieda District Development Plan.

[8] Amooti-Kaguna, B. and Nuwaha. (2000). Factors influencing choice of delivery sites in Rakai district of Uganda, Social Science and Medicine, 50, 203-213.

[9] Erlindawati, Chompikul, J. and Isaranurug, S. (2008). Factors related to the utilization of antenatal care services among women at Aceh Darussalam Province, Indonesia. Journal of Public Health Development, 6, 99-108.

[10] Kenthongkham, K. (2007). Knowledge, Attitude and Antenatal Care Practices of Women at Ad Distict, Huaphane Province, Laos. Health Service System Management, 53:45-63.

[11] Yang, Y., Yoshidal, Y., Rashid, H. and Sakamoto, J. (2010). Factors Affecting the Utilization of Antenatal Care Services Among Women in Kham District, Xiengkhouang Province. Journal of Medical Science, 72, 23-33.

[12] UNFPA. (2004a). State of the World's Population. The Cairo Consensus at Ten: Population, Reproductive Health and the Global Effort to End Poverty, 2004. New York: United Nations Population Fund.

[13] Celik, Y. and Hotchkiss, D. R. (2000). The socioeconomic determinants of maternal health care utilization in Turkey. Social Science Medicine, 50(12), 1797-1806.

[14] Magadi, M. A., Madise, N. J. and Rodrigues, R. N. (2000). Frequency and timing of antenatal care in Kenya: Explaining the variation between women of different communities. Social Science and Medicine, 51(4), 551-561.

[15] Sharma, S. K., Sawangdee Y. and Sirirassamee B. (2007). Access to health: women's status and utilization of maternal health services in Nepal. Journal of Biosocial Science, 39, 671-692.

[16] Bloom, S. S., Wypij, D. and Gupta, M. D. (2001). Dimension of women autonomy and influence on maternal health care utilization in North India City.

[17] Ngula, A. K. (2005). Women's perception on the under utilization of intrapartum care services in Okakarara district, Namibia., University of Cape Town.

[18] Chakraborty, N., Islam, M. A., Chowdbury, R. I. W. and Akhter, H. H. (2003). Determinants of the use of maternal health services in rural Bangladesh. Health Promotion International, 18(4), 327337.

[19] Fotso, J. C., Ochako, R. A. and Ezeh, A. (2006). Interplay between women's percieved quality of, and access to care and household wealth on the utilization of maternity services among the urban poor.

[20] ILO. (2003). Statistic of Employment, Unemployment, Underemployment: Economically Active Population, [Electronic Version]. Retrieved 23rd June, 2011

[21] Swenson I. E., Thang N. M., Nahn V. O. and Tieu P. X. (1993). Factors related to the utilization of prenatal care in Vietnam. Journal of Tropical Medicine and Hygiene, 96(2), 76-85.

[22] McDonagh M. (1996). Is antenatal care effective in reducing maternal morbidity and mortality? Health Policy and Planning, 11(1), 1-15.

[23] UNFPA (2004b). Maternal mortality updates: Delivering in to good hands. New York: United Nations Population Fund. 